\title{
Neurological manifestations of dengue viral infection
}

This article was published in the following Dove Press journal:

Research and Reports in Tropical Medicine

29 October 2014

Number of times this article has been viewed

Francisco Javier CarodArtal ${ }^{1,2}$

'Neurology Department, Raigmore hospital, Inverness, UK; ${ }^{2}$ Universitat Internacional de Catalunya (UIC), Barcelona, Spain

Video abstract

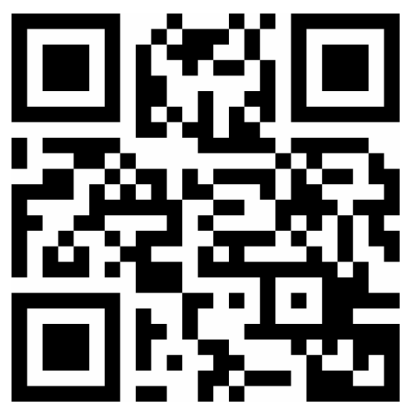

Point your SmartPhone at the code above. If you have a QR code reader the video abstract will appear. Or use: http://dvpr.es//xrafgd
Correspondence: Francisco Javier CarodArtal

Neurology Department, Raigmore Hospital, Old Perth Road, Inverness, IV2 3UJ, UK

Email javier.carodartal@nhs.net
Abstract: Dengue is the most common mosquito-borne viral infection worldwide. There is increased evidence for dengue virus neurotropism, and neurological manifestations could make part of the clinical picture of dengue virus infection in at least $0.5 \%-7.4 \%$ of symptomatic cases. Neurological complications have been classified into dengue virus encephalopathy, dengue virus encephalitis, immune-mediated syndromes (acute disseminated encephalomyelitis, myelitis, Guillain-Barré syndrome, neuritis brachialis, acute cerebellitis, and others), neuromuscular complications (hypokalemic paralysis, transient benign muscle dysfunction and myositis), and dengue-associated stroke. Common neuro-ophthalmic complications are maculopathy and retinal vasculopathy. Pathogenic mechanisms include systemic complications and metabolic disturbances resulting in encephalopathy, direct effect of the virus provoking encephalitis, and postinfectious immune mechanisms causing immune-mediated syndromes. Dengue viruses should be considered as a cause of neurological disorders in endemic regions. Standardized case definitions for specific neurological complications are still needed.

Keywords: encephalitis, encephalopathy, dengue fever, neurological complications

\section{Introduction and epidemiology}

Dengue is a mosquito-borne viral infection caused by dengue viruses (DENVs). It is the most common and the most rapidly growing mosquito-borne viral infection worldwide. ${ }^{1}$ Approximately $40 \%$ of the world population ( 2.5 billion people) may be at risk, and $75 \%$ of them are living in the Asia-Pacific region. ${ }^{1,2}$ Every year, between 50 and 100 million illnesses occur, and a half million require hospitalization. A formal modeling framework estimated that 390 million dengue infections occur every year, of which around 100 million become symptomatic and manifest apparently. ${ }^{3}$

In the last decades, a dramatic resurgence of DENV activity has occurred in the tropics, and dengue incidence has increased 30-fold. DENV are ubiquitous throughout the tropics, with local spatial variations in risk influenced by temperature, rainfall, and the degree of urbanization. ${ }^{3}$ Hyperendemicity, the cocirculation of multiple virus serotypes in an area, has been associated with the evolution of severe dengue in a geographical area. ${ }^{4}$ Factors that favored resurgence of dengue are listed in Table $1 .{ }^{1}$

In 2009, the World Health Organization (WHO) updated and categorized the new dengue definitions into dengue without warning signs, dengue with warning signs, and severe dengue (Table 2). The new dengue guidelines included the central nervous system (CNS) in the definition of severe disease. ${ }^{1}$ Indeed, "neuro-dengue" has been associated in a Brazilian study with increased mortality on suspected cases of meningitis and meningoencephalitis. ${ }^{5}$ Dengue shock syndrome and some neurological 
Table I Factors that may favor resurgence of dengue

Geographical expansion of mosquito viruses and vectors

Presence of endemic virus transmission

Hyperendemicity

Expansion from urban to semiurban and rural areas

Extensive vector infestation

Decline of vector control campaigns

Increase of human population density in urban areas

Unreliable water supply systems and poor solid waste disposal

Increase of nonbiodegradable containers in endemic areas

Human traveling

complications including dengue encephalopathy, cerebral bleeding, and Guillain-Barré syndrome (GBS) were the most frequent causes of death following dengue at a tertiary center hospital in Lahore, Pakistan. ${ }^{6}$

Although there is increased evidence of DENV neurotropism, there are no standardized case definitions or diagnostic criteria for neuro-dengue in the 2009 WHO guidelines. Recently, a classification of neurological complications of

Table 2 The 2009 World Health Organization classification for dengue severity

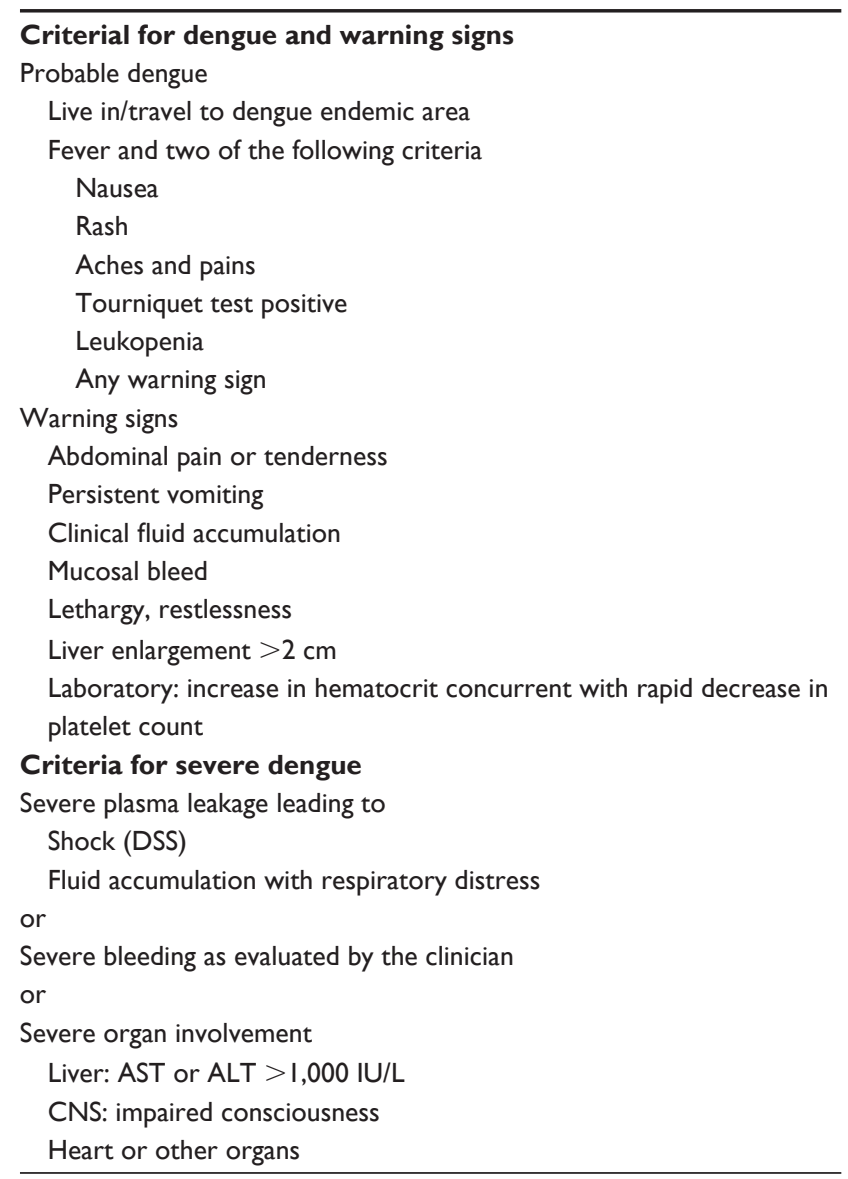

Abbreviations: ALT, alanine transaminase; AST, aspartate transaminase; CNS, central nervous system; DSS, dengue shock syndrome.
DENV infection has been proposed and categorized into dengue encephalopathy; encephalitis; immune-mediated neurological syndromes; neuromuscular, cerebrovascular, and neuro-ophthalmologic complications. ${ }^{7}$

The purpose of this article is to review the neurological manifestations of dengue viral infection and to expand and update the spectrum of neurological syndromes associated with DENV infection.

\section{Dengue viruses and vectors}

DENV are single strand RNA viruses, members of the Flaviviridae family, genus Flavivirus. The RNA genome is composed of seven nonstructural protein genes (NS) and three structural protein genes called core, membrane, and envelope, respectively. ${ }^{8}$ DENV comprise four antigenically distinct serotypes called DENV-1 to DENV-4. Several genotypes have been described from each dengue serotype and have different virulent properties. ${ }^{8}$ All four DENV serotypes can circulate simultaneously in endemic tropical and subtropical countries and in both urban and periurban environments. Epidemics are frequent following the circulation of different virus serotypes when they come into a new environment.

Except for the sylvatic cycle of dengue in rural areas of Southeast Asia and West Africa where nonhuman primates can be affected, humans are the unique reservoir hosts. ${ }^{9}$ Humans also act as an amplifying virus host during the viremic phase of the disease, and mosquitoes become infected when they bite people during the viremic phase. The extrinsic incubation period is the period between the time when the mosquito takes a viremic blood meal and the time when the mosquito becomes infectious. The duration of the extrinsic incubation period for dengue is temperature-dependent, and only at the end of this period do mosquitoes have the virus back to the salivary glands and are ready to infect and transmit the virus to other people. Once infected, the mosquito remains infective for life (30-45 days) and can transmit the virus to the progeny through transovarial transmission. ${ }^{10}$

Aedes aegypti, the main vector, has spread from Africa to other tropical and subtropical areas following international trade. A. aegypti was introduced in America following the slave trade. ${ }^{11}$ It was well established in Europe but disappeared from the Mediterranean basin in the mid-20th century for unclear reasons. The mosquito lives in the peridomestic environment, feeds preferentially on human blood, and takes several blood meals during the gonotropic cycle, so several people can be bitten in a short period of time. For all these reasons, this anthropophilic mosquito is a very efficient vector for DENV. 
Aedes albopictus, originally from Southeast Asian forests, was adapted to urban and periurban environments. ${ }^{12}$ Recently, A. albopictus has spread from Asia to other continents through the international trade of used tires, where the mosquito deposited eggs that remained viable for many months, even in absence of water collections. A. albopictus is successfully adapted to cold temperatures and has peridomestic habits but feeds also on other animals and birds; for these reasons, it can survive far from human dwellings. A. albopictus is an emergent health challenge in temperate regions. It was detected in Europe for the first time in 1979, in Albania, and spread to other European countries and the Mediterranean basin. A. albopictus has recently been incriminated in some autochthonous dengue infections in France. ${ }^{13}$

Dengue fever is the most important arboviral infection in travelers. ${ }^{14}$ People infected in an endemic area can travel within the incubation period of dengue (3-14 days) to a nonendemic place, and at that point enter the viremic phase of clinical dengue, introducing new serotypes/genotypes and contributing to the emergence of new epidemics.

The antibody-dependent enhancement hypothesis of infection has been proposed as a mechanism to explain severe dengue occurring during a secondary infection and in infants with primary infections. ${ }^{1}$ The infection with a specific dengue serotype leads the immunological system to create serotypicspecific, neutralizing antibodies. ${ }^{1}$ The immunity is lifelong, but people can suffer sequential secondary or tertiary infections depending on the circulation of different serotypes in a particular area. The immunity against a serotype gives only brief cross-protection for the other serotypes, and secondary infection with a heterologous DENV serotype can provoke a more severe disease. Nonneutralizing, cross-reactive antibodies from a previous dengue infection, or acquired passively at birth, may bind to epitopes on the surface of a heterologous infecting virus and facilitate the virus entry into Fc-receptor-bearing cells. This fact may facilitate cell invasion and enhance viremia, resulting in a higher viral burden and inducing a greater host immune response that initiates a self-amplifying cascade, leading to the release of cytokines and other proinflammatory mediators that may contribute to plasma leakage. ${ }^{1}$

\section{Neurological manifestation of dengue}

Acute dengue may present with fever; headache; pain in muscles, bones, joints, and abdominal region; and nausea and vomiting. Maculopapular rash can be observed after the third day in half of infected people. Involvement of the CNS has been described in dengue with/without warning signs and in severe dengue. Several Southeast Asian studies have shown that neurological manifestations could make up part of the clinical picture of DENV infection in around $0.5 \%-7.4 \%$ of symptomatic laboratory-confirmed cases presenting at hospital. ${ }^{7,15}$

Neurological complications of dengue infection can be categorized into dengue encephalopathy, DENV encephalitis, immune-mediated syndromes (acute disseminated encephalomyelitis [ADEM], myelitis, neuritis brachialis, and GBS, among others), dengue muscle dysfunction, dengue-associated stroke, and neuro-ophthalmic complications. $^{7}$

\section{Dengue encephalopathy}

Encephalopathy is a clinical syndrome of diminished level of consciousness. It is probably the most commonly reported neurological disturbance associated with DENV infection. Dengue encephalopathy may result from consequences of systemic infection and can be precipitated by anoxia, cerebral edema, metabolic disturbances (such as hyponatremia), prolonged shock, systemic hemorrhage, acute liver or renal failure, or release of toxic substances. ${ }^{7}$

The true incidence of dengue encephalopathy is unknown. In a 2-year prospective case-control study performed in Vietnam, dengue-associated encephalopathy accounted for $0.5 \%$ of 5,400 serologically-confirmed patients admitted with dengue hemorrhagic fever. ${ }^{16}$ Neurological symptoms were found in $5.4 \%$ of 1,493 Thai children with dengue, and half of them presented with encephalopathy. ${ }^{17}$

The analysis of cerebrospinal fluid (CSF) is usually normal in dengue encephalopathy. ${ }^{16}$ Neuroimaging studies (computed tomography and/or magnetic resonance imaging [MRI] scan) may be normal or show diffuse cerebral edema. ${ }^{18}$ Prognosis depends on causal factors, associated comorbidities, and early supportive treatment, and mortality may be high. ${ }^{16}$

\section{Dengue encephalitis}

Neurotropism and CNS invasion in DENV infection has been demonstrated in recent years. Evidence comes from three different types of epidemiological studies: 1) those that investigated the prevalence of neurological complications in patients admitted with dengue fever, 2) studies that assessed the incidence of dengue among patients admitted with encephalitis, and 3) pathological and autopsy studies of fatal dengue. ${ }^{?}$ 
In a prospective study of 175 hospitalized dengue patients in South India, DENV encephalitis was recorded in 7.4\% of dengue cases. ${ }^{19}$ In another prospective hospital-based study of 526 acute encephalitis patients from eastern India, DENV was responsible for $0.6 \%$ of cases of encephalitis. ${ }^{20}$ In Vietnam, the $4.2 \%$ of 378 patients with suspected CNS infection were infected with DENV as compared to $1.4 \%$ of 286 matched hospital controls. ${ }^{13}$ In Brazil, a hospital case series showed that dengue was the leading cause of encephalitis in half of 47 cases. ${ }^{21}$

DENV has been found in the CSF, but not in the serum, of some patients with neurological symptoms, and this fact points against a vascular leak or rupture of the blood-brain barrier as a pathogenic mechanism of dengue encephalitis. Detection of viral antigen in brain tissue and concomitant CSF pleocytosis and DENV RNA amplification in the CSF are evidence to support the invasive neurotropism of DENV. ${ }^{?}$ Dengue viral RNA amplification has been obtained from CSF during acute infection. ${ }^{15}$

Evidence of DENV neurotropism was also found in autopsy studies. DENV antigens have been isolated from brain tissues in fatal dengue cases. ${ }^{22}$ DENV-4 was detected by immunohistochemistry and by reverse transcriptase polymerase chain reaction assay (RT-PCR) in the inferior olivary nucleus of medulla and in the granular layer of cerebellum of a fatal case of dengue hemorrhagic fever. Immunoreactivity was observed in neurons, astrocytes, microglia, and endothelial cells. ${ }^{22}$

Viral factors are also implicated in neuropathogenesis. Mutations of three amino acids in DENV-1 produced a neurovirulent phenotype that may provoke encephalitis and leptomeningitis. Amino acid substitutions were mapped to the envelope structural protein E and the nonstructural NS3 helicase domains in mice. ${ }^{23}$ Other risk factors for dengue encephalitis include DENV-2 and DENV-3 serotype infections and age, as young adults and children are at higher risk. ${ }^{15}$

Reduced level of consciousness, headache, fever, nausea and vomiting, seizures, focal neurological deficits, and behavioral symptoms can be seen in dengue encephalitis patients. ${ }^{17}$ No specific neuroimaging findings suggestive of dengue encephalitis have been reported. Brain MRI may be normal or show some focal parenchymal abnormalities. ${ }^{16,24}$ Symmetric gyral edema and altered signal intensity involving bilateral temporal perisylvian regions, hippocampi, and cingulated gyri have been reported. ${ }^{25}$ The thalamus, pons, and bilateral cerebellum cortex can also be involved. ${ }^{26}$ Meningeal enhancement on postcontrast MRI has been seen occasionally. ${ }^{18}$

\section{Immune-mediated neurological syndromes}

Immune-mediated neurological syndromes have been reported following DENV infection. They include mononeuropathies, GBS, brachial neuritis, transverse myelitis, ADEM, acute cerebellitis, opsoclonus-myoclonus, and parkinsonism. ${ }^{7}$ These postinfectious syndromes have been linked temporally to DENV infection, and a causal association has been inferred.

An immune-mediated pathogenic mechanism has been proposed following DENV infection since the virus has not been isolated in the CSF of these patients. Indeed, DENV can trigger cytokine overproduction resulting in immunemediated dysfunction of endothelial cells.

\section{Mononeuropathies}

Involvement of cranial nerves following dengue fever has been reported. Optic neuritis, oculomotor nerve palsy, isolated sixth nerve palsy, isolated Bell's palsy, long thoracic neuropathy, and isolated phrenic nerve palsy causing diaphragmatic paralysis have been described. ${ }^{27-35}$

\section{Polyradiculoneuritis and GBS}

Cases of acute polyradiculoneuritis, lumbosacral plexopathy, GBS, and Miller Fisher syndrome have been reported following DENV infection. ${ }^{36-42}$ The clinical picture of dengueassociated GBS consists of acute and progressive weakness affecting distal-to-proximal muscles in both lower and upper limbs. Autonomic symptoms are also common and may occur in one third of patients. Generalized hypo- or areflexia is noted on examination. Albuminocytological dissociation with increased protein levels and absence of pleocytosis are found in the CSF. Sensory and motor velocity conduction tests may be helpful to differentiate GBS from dengue-associated hypokalemic paralysis. ${ }^{43}$ The pure axonal motor-sensory variant of GBS has also been reported. ${ }^{41}$

Cases of brachial neuritis and neuralgic amyotrophy syndrome associated with dengue have been reported. Patients presented with acute onset of weakness in an upper limb, preceded by moderate neuropathic pain in the shoulder region. Symptoms usually started around 2 weeks after resolving viral infection. On exam, weakness and atrophy of the proximal musculature of the upper limb was detected. ${ }^{44}$ Neurophysiological examinations may reveal axonal changes $(70 \%)$ or demyelinating abnormalities $(30 \%){ }^{45}$

\section{Acute transverse myelitis}

Myelitis following DENV infection can occur during either the parainfectious or the postinfectious stages of 
dengue infection. ${ }^{46-52}$ Direct virus invasion and immunemediated factors are pathogenic mechanisms occurring in each one of these stages, respectively.

Acute transverse myelitis has been reported following DENV infection. ${ }^{46,47}$ Severe paraparesis and even paraplegia, neurogenic pain, sensory symptoms, and neurogenic bladder (urinary retention and incontinence) may happen. Postinfectious myelitis usually occurs between 1 and 2 weeks after the onset of initial symptoms, whereas parainfectious myelitis may happen within the first week of infection. ${ }^{48,49}$

Intrathecal synthesis of anti-dengue specific immunoglobulin $\mathrm{G}(\mathrm{IgG})$ antibodies have been detected in some patients with dengue myelitis. ${ }^{50} \mathrm{~T} 2$-weighted MRI may reveal extensive high-intensity signal throughout the lower cervical to upper lumbar regions in the spinal cord. ${ }^{27}$ Nevertheless, spinal cord MRI may be normal in some patients. ${ }^{46}$

Neuromyelitis optica following DENV infection is uncommon. ${ }^{53}$ Multiple high-intensity spotty lesions were found in the spinal cord MRI in one patient, whereas images of the brain revealed no abnormalities. Myelopathy associated to spinal epidural hematoma and brainstem hemorrhage is another unusual complication of DENV infection. ${ }^{54}$

\section{Acute disseminated encephalomyelitis}

ADEM is an immune-mediated syndrome that may occur during the convalescence phase following DENV infection and dengue hemorrhagic fever. ${ }^{37,55-61}$ Neurological symptoms include seizures, altered sensorium, and focal neurological deficits; they may occur following the hyperpyrexic phase of dengue. .7,58 $^{57}$

Mild pleocytosis and moderate rise in protein concentration can be detected in the CSF. Brain MRI may show white matter lesions on T2-weighted images in the centrum semiovale, corona radiate, corpus callosum, and thalamus. Spinal cord abnormalities may be seen mainly in the thoracic and cervical regions. ${ }^{18}$

\section{Acute cerebellitis and other autoimmune syndromes}

Acute cerebellitis following DENV infection has been recently described. ${ }^{60}$ The clinical picture consists of dysarthria, horizontal nystagmus, bilateral dysmetria, dysdiadochokinesia, incoordination and wide-based ataxic gait. The presence of anti-DENV immunoglobulin M (IgM) in blood and CSF confirmed the diagnosis. MRI of brain was performed in some patients and was normal. ${ }^{61}$ Most patients had a self-limiting course with complete recovery, and clinical symptoms spontaneously resolved within $2-3$ weeks after dengue infection.
Opsoclonus myoclonus syndrome has been described in two patients who suffered dengue. Clinical symptoms included erratic and involuntary conjugate ocular saccades (opsoclonus), axial myoclonus, gait ataxia, and other cerebellar signs. The CSF analysis and the brain MRI did not reveal any abnormalities. ${ }^{45} \mathrm{~A}$ case of parkinsonism following dengue fever has been reported in one patient. Positive oligoclonal bands were detected in the CSF. ${ }^{62}$

\section{Neuromuscular complications}

DENV infection may lead to acute neuromuscular weakness because of hypokalemia, myositis, and GBS. ${ }^{63}$ Three different pathogenic mechanisms may explain acute neuromuscular weakness following dengue infection. Dengue-associated hypokalemic paralysis can be considered as a systemic complication of dengue infection, whereas myositis could be related to viral neurotropism and/or immune factors. Finally, GBS would be an immune-mediated syndrome following dengue.

\section{Dengue transient muscle dysfunction}

The muscle system is commonly affected during DENV infection. Clinical symptoms are usually self-limiting and may vary from mild myalgia to severe myositis and even rhabdomyolysis. Clinical case series of dengue patients reported elevated creatinine phosphokinase (CPK) levels in most of them, myalgia in $63 \%$, and mild muscle weakness in around 3\%. ${ }^{64-70}$ The term "dengue associated transient muscle dysfunction" is now preferred to dengue myositis due to the benign self-limiting nature.

A varying degree of transient myalgia lasting a few days, muscle tenderness on stretching, proximal muscle weakness, hypotonia, and elevated muscle enzymes can be found. ${ }^{66,67}$ Spontaneous recovery is expected in most patients within 1-2 weeks. Children may present with a sudden onset of calf pain and difficulty in walking. ${ }^{68}$ Intense muscle pain; severe quadriparesis; neck, trunk, and respiratory muscle weakness; and rhabdomyolysis may occur in the more severe cases. ${ }^{67,69,70}$ Acute flaccid paralysis can happen with or without myalgia.

CPK levels are usually elevated during the first week of illness and may range from $500 \mathrm{IU} / \mathrm{L}$ to more than 100,000 IU/L. ${ }^{67}$ The higher CPK levels, the more intense the muscle inflammation. Simultaneous occurrence of myositis and myocarditis may happen in some patients, and raised levels of muscle and brain isoenzyme of creatine kinase index suggests myocardial involvement. ${ }^{71}$

Electromyography may reveal early recruitment of motor unit action potentials (MUAPs) with normal MUAP 
morphology and no spontaneous activity. ${ }^{65,67}$ Sensory and motor velocity conduction tests are usually normal, and this may be helpful in the differential diagnosis of GBS.

Muscle biopsy may reveal extensive interstitial hemorrhage splaying separate from the myofibers. ${ }^{65}$ In mild cases, a moderate perivascular mononuclear infiltrate and lipid accumulation were found. Perivascular inflammation, myonecrosis, increase in the number of mitochondria, and fiber type grouping can be observed during the prodromal phase. ${ }^{65,69}$

Dengue transitory muscle dysfunction has been attributed to changes in vascular permeability, whereas severe dengue myositis may be secondary to direct viral invasion of the muscle and immune-mediated damage of muscle fibers. ${ }^{65}$ The presence of metabolic alterations, edema, and hemorrhage may be responsible for the transient muscle weakness. Myotubes can also be infected by DENV and have an increased expression of inflammatory genes and protein IP-10 and rise of intracellular $\mathrm{Ca}^{2+}$ concentration. ${ }^{72}$ Host and viral factors are probably responsible for the varying severity of muscle symptoms.

\section{Dengue-associated hypokalemic paralysis}

In a case series of 29 patients, DENV infection was the second leading cause of hypokalemic paralysis, after thyrotoxicosis. ${ }^{43}$ Dengue-associated hypokalemic paralysis may present with weakness, usually in the stage of defervescence and not at the peak of febrile episode. Clinical symptoms include severe weakness and quadriparesis. Motor weakness, hypotonia, hyporeflexia, flexor plantar reflexes, and indemnity of sensory function can be seen on neurological examination. ${ }^{73-75}$ Potassium levels may range between 1.0 and $3.5 \mathrm{mEq} / \mathrm{L}$, whereas CPK levels are usually mildly elevated. Low compound MUAPs coupled with robust sensory nerve action potentials were observed during the period of maximum weakness.

Differential diagnosis includes thyrotoxic paralysis, channelopathies, dengue-associated myositis, and GBS. Physicians should be aware of this systemic complication of dengue infection, as it is potentially treatable even with low doses of potassium. Indeed, a rapid recovery has been noted in treated patients.

\section{Dengue-associated stroke}

Stroke is a severe complication of DENV infection. It may occur during the convalescence period and can be potentially fatal. ${ }^{76,77}$ Both ischemic and hemorrhagic strokes have been associated with DENV infection, although the latter is much more common (Table 3). ${ }^{7}$ The proportion of hospitalized, confirmed dengue patients who suffer a hemorrhagic stroke may range from $0.26 \%$ (India) to $0.06 \%$ (Brazil). ${ }^{78,79}$

Clinical presentation includes fever, moderate to severe headache, vomiting, acute hemiparesis, and loss of consciousness. Many patients have no spontaneous visible bleeding from other sites except intracranial bleeding. ${ }^{76}$ Although common hemorrhagic features in severe dengue include ecchymosis, petechial hemorrhage, epistaxis, and gastrointestinal bleed, acute intracranial bleeding can occur in absence of these systemic hemorrhagic manifestations. Most patients suffered brain hemorrhage 1 week after the onset of fever. Basal ganglia hemorrhage and multiple hemorrhagic foci in brain lobes are the most common stroke subtypes. Large intracranial bleeding can be complicated with edema, mass effect, and hydrocephalus. Dengue-associated ischemic stroke has also been described..$^{77,80,81}$

Increased vascular permeability, plasma leakage, vasculitis, prolonged prothrombin time, severe thrombocytopenia, hepatic dysfunction, and mild degree of disseminated intravascular coagulation may contribute to the pathogenesis of dengue-associated stroke. ${ }^{82}$

\section{Neuro-ophthalmic complications}

Neuro-ophthalmic manifestations of DENV infection include dengue maculopathy, retinal hemorrhages, optic disc swelling, and optic neuropathy. ${ }^{28,83-85}$ They have been described in dengue with/without warning symptoms and in severe dengue cases. ${ }^{86}$ Clinical symptoms include blurred vision and central escotoma and may occur during the convalescent stage.

Retinal vasculopathy is a common ophthalmic complication of dengue infection, and retinal hemorrhages may be a further problem associated with increased vascular permeability and breakdown of the inner blood-retinal barrier. ${ }^{84}$ Microinfarctions of the nerve fiber layer due to occlusion of precapillary arterioles can be detected as cotton-wool spots on ophthalmologic examination. ${ }^{85} \mathrm{Immu}-$

Table 3 Dengue-associated stroke subtypes

Hemorrhagic stroke
Basal ganglia hemorrhage
Brain lobe hemorrhage (single or multiple)
Bilateral cerebellar hemorrhage
Pontine hemorrhage
Acute subdural hematoma (single or multiple)
Pituitary adenoma hemorrhage
Subarachnoid hemorrhage
Ischemic stroke
Watershed infarct
Cortical infarcts
Lacunar infarcts in corona radiata and putamen


nological activation response and the release of vasoactive and procoagulant cytokines would be responsible for retinal vasculopathy.

Dengue maculopathy is characterized by macular swelling, yellow spots in the macula, and macular hemorrhages. Exudative maculopathy and small hemorrhages located in the optic nerve fiber layer can provoke a reduced visual acuity. Prevalence of dengue maculopathy among hospitalized, confirmed dengue patients has been estimated in $10 \% .{ }^{87}$

Fundus fluorescein and indocyanine green angiography (FFA), optical coherence tomography, and visual field testing are useful tools in the diagnosis of neuro-ophthalmic dengue. ${ }^{86}$ FFA may show leakage of the optic disc and retinal vessels, retinal pigment epithelium defects, and macular nonperfusion. ${ }^{84}$ Blocked fluorescence, venular occlusion, and venular leakage can be found on FFA in dengue maculopathy. Optical coherence tomography may show diverse patterns of maculopathy including diffuse retinal thickening, cystoid macular edema, and foveolitis. ${ }^{86}$

\section{Diagnosis}

A wide variety of viral and bacterial infections can affect the CNS and provoke a similar clinical picture to neurodengue in the tropics. Differential diagnosis is summarized in Table 4.

DENV can be detected in serum or blood samples during early days of infection. The best methods for rapid diagnosis at that time are viral culture and detection of NS1 antigen and RNA. ${ }^{1}$ Culture may not be a useful method due to its cost and the complex technical requirements. Molecular diagnosis also requires technical expertise but is more feasible than virus culture. Several RT-PCR techniques (nested RT-PCR, real time RT-PCR, and multiplex RT-PCR) have been developed and are helpful to detect all four virus serotypes. RT-PCR is highly sensitive and specific and is also useful for phylogenetic analysis of DENV. ${ }^{8}$ Several enzyme-linked

Table 4 Differential diagnosis of a febrile encephalopathy/ encephalitis in the tropics

Cerebral malaria

Leptospirosis

Typhoid fever

Rickettsial infection

Tuberculosis meningitis

Japanese encephalitis virus

West Nile virus infection

Herpes simplex virus infection

Enteroviruses infection

Other viral and bacterial infections

Dengue encephalopathy immunosorbent assays (ELISAs) for dengue NS1 antigen detection have been developed. ${ }^{88} \mathrm{NS} 1$ antigen is detectable in the first days of infection, even before detecting IgM antibodies.

Antibodies can be detected in serum samples between 3 and 10 days after symptoms onset. IgM-specific capture ELISA (MAC-ELISA), complement fixation, and hemagglutination inhibition tests can identify host immune response. The MAC-ELISA test is the most widely used. The IgG antibodies detection test when properly used in paired serum samples collected during the acute and convalescent phases can differentiate primary from secondary dengue infections. ${ }^{89}$ Dengue IgM appears in serum 5-10 days from the beginning of infection and, in case of secondary infection, achieves low levels.

Diagnosis can be improved when using both NS1 antigen and IgM antibodies instead of using antigen tests or antibodies alone..$^{90} \mathrm{~A}$ recent test combining the detection of NS1 antigen and anti-NS1 IgM was more sensitive that RT-PCR alone. The detection of NS1 antigen by immunochromatographic test has $87.5 \%$ sensitivity and may be a faster and cheaper diagnostic technique than PCR. ${ }^{91}$ The use of PCR and/or immunoassay techniques is recommended during the first week of fever, whereas the MAC-ELISA test should be used in patients with more than 5-7 days of fever.

CSF analysis may be helpful to differentiate dengue encephalitis from encephalopathy. Lymphocytic pleocytosis and mild increase in CSF protein can be found in the CSF of patients with DENV encephalitis; however, these findings are not always present, and normal CSF cellularity should not exclude dengue encephalitis. ${ }^{24}$ Detection of dengue NS1 antigen, DENV, and DENV-specific IgM antibodies in the CSF are helpful to confirm the diagnosis of dengue encephalitis. ${ }^{26,92}$

However, sensitivity of immunological techniques can be low, and DENV-specific IgM antibodies have been detected in only one third of dengue encephalitis patients. ${ }^{15,93}$ IgM antibody measurement may not be a reliable diagnostic marker of neuro-dengue due to low titers detected in the CSF. RT-PCR also has lower sensitivity in CSF as compared to serum due to a lower CSF viral load.

According to the WHO case definition, confirmed dengue is defined by one of the following: PCR-positive; virus culture positive; IgM seroconversion in paired serum samples; or four times IgG titer increase in paired serum samples. ${ }^{2}$

\section{Management}

A good clinical suspicion and the early detection of warning symptoms are essential to efficiently manage patients with 
dengue fever. There are no effective antiviral drugs available for the treatment of DENV infection. Mild cases of dengue fever may be managed on a domiciliary basis. Oral rehydration therapy, healthy food, rest, and antipyretic drugs such as paracetamol are useful to treat mild dengue cases. Nonsteroid anti-inflammatory drugs and acetylsalicylic derivatives should be avoided. ${ }^{2}$

Severe dengue can be complicated with hemorrhagic diathesis, vascular leakage, and hypovolemic shock. Early recognition of this severe complication and monitoring for drop of platelet count and rise of hematocrit is recommended. Shock can be prevented by early volume replacement with isotonic crystalloid solutions. Colloid isotonic solutions should be given to patients who fail initial therapy or present profound shock. The use of oral steroids is not helpful to prevent shock or other systemic complications of DENV infection. Platelet transfusion has been indicated when the platelet level drops significantly below 20,000 and a risk of bleeding is detected, or when the patient has hemorrhages and the platelet count is $21,000-40,000 .^{2}$

In patients suffering dengue encephalopathy, underlying metabolic causes such as liver dysfunction, hyponatremia, metabolic acidosis, or hypokalemia should be recognized and corrected early. Management of DENV encephalitis remains supportive and adequate hydration, nutrition, maintenance of airway, and monitoring of consciousness level are needed. There is no effective viral therapy to treat dengue encephalitis. Associated-dengue stroke should be treated according to established therapeutic guidelines for stroke. High doses of intravenous immunoglobulins may be helpful to treat GBS following dengue infection. Steroids (intravenous methylprednisolone pulses, oral prednisolone) have been advocated as therapy to treat dengue immune-mediated neurological complications such as ADEM, transverse myelitis, brachial neuritis, or retinal vasculitis. Nevertheless, no clinical trials have evaluated either the safety and efficacy of steroids or the optimal dose and route of administration. Hydration, resting, and analgesic drugs may be helpful to treat myalgia and transitory muscle dysfunction. Early rehabilitation is advised for those severe neurological complications including myelitis. ${ }^{7}$

There is no vaccine available against DENV, but live, attenuated, tetravalent dengue vaccines are being studied in ongoing research programs. An effective vaccine should provide protective immunity against all dengue serotypes without antibody-dependent enhancement. Results from a Phase IIb trial with a tetravalent live-attenuated chimeric vaccine were disappointing and showed an efficacy of 30\% against all four overall, with no protection against DENV-2. ${ }^{94}$
Control of dengue infection depends on the efforts to control insect vector, and a major control measure is to reduce either the number of larva in containers or the number of larval habitats. Several vector control strategies have been implemented in some tropical countries, including biological and chemical control and environmental and vector management. Interventions using a community-based integrated approach are needed.

\section{Conclusion}

Dengue neurotropism is still a controversial topic. However, DENV can cause neurological manifestations more commonly than previously thought and may represent a significant underreported cause of viral encephalitis in the tropics. ${ }^{3,7}$ In the literature, the terms "encephalopathy" and "encephalitis" have been used interchangeably. For this reason, a correct interpretation of neurological signs and symptoms, CSF analysis, and MRI of brain and spinal cord are needed to categorize neuro-dengue. In poor, developing countries with limited access to medical resources, neuroimaging techniques and immunological CSF studies are limited; for this reason, clinical classification of neurological syndromes following DENV infection may be limited. A standard definition to distinguish dengue encephalitis from dengue encephalopathy and other neurological disturbances is needed.

\section{Disclosure}

No funding sources have supported this research. The author reports no conflicts of interest in this work.

\section{References}

1. Chakravarti A, Arora R, Luxemburger C. Fifty years of dengue in India. Trans R Soc Trop Med Hyg. 2012;106(5):273-282.

2. World Health Organization. Dengue guidelines for diagnosis, treatment, prevention and control. Geneva, Switzerland: WHO, 2009.

3. Bhatt S, Gething PW, Brady OJ, et al. The global distribution and burden of dengue. Nature. 2013;496(7446):504-507.

4. Murray NE, Quam MB, Wilder-Smith A. Epidemiology of dengue: past, present and future prospects. Clin Epidemiol. 2013;5:299-309.

5. Araújo FM, Araújo MS, Nogueira RM, et al. Central nervous system involvement in dengue: a study in fatal cases from a dengue endemic area. Neurology. 2012;78(10):736-742.

6. Assir MZ, Ahmad HI, Masood MA, Kamran U, Yusuf NW. Deaths due to dengue fever at a tertiary care hospital in Lahore, Pakistan. Scand $J$ Infect Dis. 2014;46(4):303-309.

7. Carod-Artal FJ, Wichmann O, Farrar J, Gascón J. Neurological complications of dengue virus infection. Lancet Neurol. 2013;12(9):906-919.

8. Rico-Hesse R. Dengue virus evolution and virulence models. Clin Infect Dis. 2007;44(11):1462-1466.

9. Chen R, Vasilakis N. Dengue - quo tu et quo vadis? Viruses. 2011;3(9): $1562-1608$.

10. Thongrungkiat S, Maneekan P, Wasinpiyamongkol L, Prummongkol S. Prospective field study of transovarial dengue-virus transmission by two different forms of Aedes aegypti in an urban area of Bangkok, Thailand. J Vector Ecol. 2011;36(1):147-152. 
11. Reiter P. Yellow fever and dengue: a threat to Europe? Euro Surveill. 2010;15(10):19509.

12. Paupy C, Delatte H, Bagny L, Corbel V, Fontenille D. Aedes albopictus, an arbovirus vector: from the darkness to the light. Microbes Infect. 2009;11(14-15):1177-1185.

13. Gould EA, Gallian P, De Lamballerie X, Charrel RN. First cases of autochthonous dengue fever and chikungunya fever in France: from bad dream to reality! Clin Microbiol Infect. 2010;16(12):1702-1704.

14. Wichmann O, Gascon J, Schunk M, et al; European Network on Surveillance of Imported Infectious Diseases. Severe dengue virus infection in travelers: risk factors and laboratory indicators. $J$ Infect Dis. 2007;195(8):1089-1096.

15. Solomon T, Dung NM, Vaughn DW, et al. Neurological manifestations of dengue infection. Lancet. 2000;355(9209):1053-1059.

16. Cam BV, Fonsmark L, Hue NB, Phuong NT, Poulsen A, Heegaard ED. Prospective case-control study of encephalopathy in children with dengue hemorrhagic fever. Am J Trop Med Hyg. 2001;65(6):848-851.

17. Pancharoen C, Thisyakorn U. Neurological manifestations in dengue patients. Southeast Asian J Trop Med Public Health. 2001;32(2): 341-345.

18. Wasay M, Channa R, Jumani M, Shabbir G, Azeemuddin M, Zafar A. Encephalitis and myelitis associated with dengue viral infection clinical and neuroimaging features. Clin Neurol Neurosurg. 2008;110(6):635-640.

19. Neeraja M, Lakshmi V, Teja VD, et al. Unusual and rare manifestations of dengue during a dengue outbreak in a tertiary care hospital in South India. Arch Virol. 2014;159(7):1567-1573.

20. Rathore SK, Dwibedi B, Kar SK, Dixit S, Sabat J, Panda M. Viral aetiology and clinico-epidemiological features of acute encephalitis syndrome in eastern India. Epidemiol Infect. 2014:1-8. [Epub ahead of print].

21. Soares CN, Cabral-Castro MJ, Peralta JM, de Freitas MR, Zalis M, Puccioni-Sohler M. Review of the etiologies of viral meningitis and encephalitis in a dengue endemic region. J Neurol Sci. 2011;303(1-2): 75-79.

22. Ramos C, Sánchez G, Pando RH, et al. Dengue virus in the brain of a fatal case of hemorrhagic dengue fever. J Neurovirol. 1998;4(4):465-468.

23. Bordignon J, Strottmann DM, Mosimann AL, et al. Dengue neurovirulence in mice: identification of molecular signatures in the E and NS3 helicase domains. J Med Virol. 2007;79(10):1506-1517.

24. Misra UK, Kalita J, Syam UK, Dhole TN. Neurological manifestations of dengue virus infection. J Neurol Sci. 2006;244(1-2):117-122.

25. Muzaffar J, Venkata Krishnan P, Gupta N, Kar P. Dengue encephalitis: why we need to identify this entity in a dengue-prone region. Singapore Med J. 2006;47(11):975-977.

26. Borawake K, Prayag P, Wagh A, Dole S. Dengue encephalitis. Indian J Crit Care Med. 2011;15(3):190-193.

27. Weeratunga PN, Caldera MC, Gooneratne IK, Gamage R, Perera P. Neurological manifestations of dengue: a cross sectional study. Travel Med Infect Dis. 2014;12(2):189-193.

28. Sanjay S, Wagle AM, Au Eong KG. Optic neuropathy associated with dengue fever. Eye (Lond). 2008;22(5):722-724.

29. Donnio A, Béral L, Olindo S, Cabie A, Merle H. [Dengue, a new etiology in oculomotor paralysis]. Can J Ophthalmol. 2010;45(2):183-184. French.

30. Shivanthan MC, Ratnayake EC, Wijesiriwardena BC, Somaratna KC, Gamagedara LK. Paralytic squint due to abducens nerve palsy: a rare consequence of dengue fever. BMC Infect Dis. 2012;12:156.

31. Peter S, Malhotra N, Peter P, Sood R. Isolated Bell's palsy - an unusual presentation of dengue infection. Asian Pac J Trop Med. 2013;6(1): $82-84$.

32. Chappuis F, Justafré JC, Duchunstang L, Loutan L, Taylor WR. Dengue fever and long thoracic nerve palsy in a traveler returning from Thailand. J Travel Med. 2004;11(2):112-114.

33. Chien J, Ong A, Low SY. An unusual complication of dengue infection. Singapore Med J. 2008;49(12):e340-e342.

34. Ansari MK, Jha S, Nath A. Unilateral diaphragmatic paralysis following dengue infection. Neurol India. 2010;58(4):596-598.
35. Ratnayake EC, Shivanthan C, Wijesiriwardena BC. Diaphragmatic paralysis: a rare consequence of dengue fever. BMC Infect Dis. 2012;12:46.

36. Koshy JM, Joseph DM, John M, et al. Spectrum of neurological manifestations in dengue virus infection in Northwest India. Trop Doct. 2012;42(4):191-194.

37. Palma-da Cunha-Matta A, Soares-Moreno SA, Cardoso-de Almeida A, Aquilera-de Freitas V, Carod-Artal FJ. [Neurological complications arising from dengue virus infection]. Rev Neurol. 2004;39(3):233-237. Spanish.

38. Oehler E, Le Henaff O, Larre P, Ghawche F. [Guillain-Barré syndrome following type 4 dengue in Polynesia]. Med Trop (Mars). 2011;71(2):203-204. French.

39. Jackson ST, Mullings A, Bennett F, Khan C, Gordon-Strachan G, Rhoden T. Dengue infection in patients presenting with neurological manifestations in a dengue endemic population. West Indian Med J. 2008;57(4):373-376.

40. Chen TY, Lee CT. Guillain-Barré syndrome following dengue fever. Ann Emerg Med. 2007;50(1):94-95.

41. Kumar S, Prabhakar S. Guillain-Barre syndrome occurring in the course of dengue fever. Neurol India. 2005;53(2):250-251.

42. Gaultier C, Angibaud G, Laille M, Lacassin F. [Probable Miller Fisher syndrome during Dengue fever type 2]. Rev Neurol (Paris). 2000;156(2):169-171. French.

43. Garg RK, Malhotra HS, Verma R, Sharma P, Singh MK. Etiological spectrum of hypokalemic paralysis: A retrospective analysis of 29 patients. Ann Indian Acad Neurol. 2013;16(3):365-370.

44. Verma R, Sharma P, Khurana N, Sharma LN. Neuralgic amyotrophy associated with dengue fever: case series of three patients. J Postgrad Med. 2011;57(4):329-331.

45. Verma R, Sharma P, Garg RK, Atam V, Singh MK, Mehrotra HS. Neurological complications of dengue fever: experience from a tertiary center of north India. Ann Indian Acad Neurol. 2011;14(4):272-278.

46. Seet RC, Lim EC, Wilder-Smith EP. Acute transverse myelitis following dengue virus infection. J Clin Virol. 2006;35(3):310-312.

47. Chanthamat N, Sathirapanya P. Acute transverse myelitis associated with dengue viral infection. J Spinal Cord Med. 2010;33(4):425-427.

48. Leão RN, Oikawa T, Rosa ES, et al. Isolation of dengue 2 virus from a patient with central nervous system involvement (transverse myelitis). Rev Soc Bras Med Trop. 2002;35(4):401-404.

49. Kunishige M, Mitsui T, Tan BH, et al. Preferential gray matter involvement in dengue myelitis. Neurology. 2004;63(10):1980-1981.

50. Puccioni-Sohler M, Soares CN, Papaiz-Alvarenga R, Castro MJ, Faria LC, Peralta JM. Neurologic dengue manifestations associated with intrathecal specific immune response. Neurology. 2009;73(17): 1413-1417.

51. Singh P, Joseph B. Paraplegia in a patient with dengue. Neurol India. 2010;58(6):962-963.

52. Larik A, Chiong Y, Lee LC, Ng YS. Longitudinally extensive transverse myelitis associated with dengue fever. BMJ Case Rep. 2012; May 11: 2012

53. Miranda de Sousa A, Puccioni-Sohler M, Dias Borges A, Fernandes Adorno L, Papais Alvarenga M, Papais Alvarenga RM. Post-dengue neuromyelitis optica: case report of a Japanese-descendent Brazilian child. J Infect Chemother. 2006;12(6):396-398.

54. Singh M, Garg K, Bisht A, et al. Spinal epidural hematoma with myelitis and brainstem hemorrhage: an unusual complication of dengue fever. Neurol India. 2013;61(5):541-543.

55. Brito CA, Sobreira S, Cordeiro MT, Lucena-Silva N. Acute disseminated encephalomyelitis in classic dengue. Rev Soc Bras Med Trop. 2007;40(2):236-238.

56. Chowdhury RN, Siddiqui MR, Mahbub MS, et al. Dengue fever as a cause of acute disseminated encephalomyelitis (ADEM). $J$ Medicine. 2011;12(2):185-187.

57. Karoli R, Siddiqi Z, Fatima J, Maini S. Was it a case of acute disseminated encephalomyelitis? A rare association following dengue fever. J Neurosci Rural Pract. 2013;4(3):318-321. 
58. Gupta M, Nayak R, Khwaja GA, Chowdhury D. Acute disseminated encephalomyelitis associated with dengue infection: a case report with literature review. J Neurol Sci. 2013;335(1-2):216-218.

59. Sundaram C, Uppin SG, Dakshinamurthy KV, Borgahain R. Acute disseminated encephalomyelitis following dengue hemorrhagic fever. Neurol India. 2010;58(4):599-601.

60. Weeratunga PN, Caldera HP, Gooneratne IK, et al. Spontaneously resolving cerebellar syndrome as a sequelae of dengue viral infection: a case series from Sri Lanka. Pract Neurol. 2014;14(3):176-178.

61. Withana M, Rodrigo C, Chang T, Karunanayake P, Rajapakse S. Dengue fever presenting with acute cerebellitis: a case report. BMC Res Notes. 2014;7:125.

62. Azmin S, Sahathevan R, Suehazlyn Z, et al. Post-dengue parkinsonism. BMC Infect Dis. 2013;13:179.

63. Hira HS, Kaur A, Shukla A. Acute neuromuscular weakness associated with dengue infection. J Neurosci Rural Pract. 2012;3(1):36-39.

64. Said SM, Elsaeed KM, Zakareya A. Benign acute myositis in association with acute dengue viruses' infections. Egypt J Neurol Psychiat Neurosurg. 2008;45:193-200.

65. Misra UK, Kalita J, Maurya PK, Kumar P, Shankar SK, Mahadevan A. Dengue-associated transient muscle dysfunction: clinical, electromyography and histopathological changes. Infection. 2012;40(2):125-130.

66. Lim M, Goh HK. Rhabdomyolysis following dengue virus infection. Singapore Med J. 2005;46(11):645-646.

67. Paliwal VK, Garg RK, Juyal R, et al. Acute dengue virus myositis: a report of seven patients of varying clinical severity including two cases with severe fulminant myositis. J Neurol Sci. 2011;300(1-2):14-18.

68. Ahmad R, Abdul Latiff AK, Abdul Razak S. Myalgia Cruris Epidemica: an unusual presentation of dengue fever. Southeast Asian J Trop Med Public Health. 2007;38(6):1084-1087.

69. Acharya S, Shukla S, Mahajan SN, Diwan SK. Acute dengue myositis with rhabdomyolysis and acute renal failure. Ann Indian Acad Neurol. 2010;13(3):221-222.

70. Kalita J, Misra UK, Mahadevan A, Shankar SK. Acute pure motor quadriplegia: is it dengue myositis? Electromyogr Clin Neurophysiol. 2005;45(6):357-361.

71. Sangle SA, Dasgupta A, Ratnalikar SD, Kulkarni RV. Dengue myositis and myocarditis. Neurol India. 2010;58(4):598-599.

72. Salgado DM, Eltit JM, Mansfield K, et al. Heart and skeletal muscle are targets of dengue virus infection. Pediatr Infect Dis J. 2010;29(3): 238-242.

73. Jain RS, Handa R, Prakash S, Nagpal K, Gupta P. Acute hypokalemic quadriparesis: an atypical neurological manifestation of dengue virus. J Neurovirol. 2014;20(1):103-104.

74. Jha S, Ansari MK. Dengue infection causing acute hypokalemic quadriparesis. Neurol India. 2010;58(4):592-594.

75. Gutch M, Agarwal A, Amar A. Hypokalemic quadriparesis: an unusual manifestation of dengue fever. J Nat Sci Biol Med. 2012;3(1):81-83.

76. Kumar R, Prakash O, Sharma BS. Intracranial hemorrhage in dengue fever: management and outcome: a series of 5 cases and review of literature. Surg Neurol. 2009;72(4):429-433; discussion 433.
77. Verma R, Sahu R, Singh AS, Atam V. Dengue infection presenting as ischemic stroke: an uncommon neurological manifestation. Neurol India. 2013;61(3):317-318.

78. Mathew S, Pandian JD. Stroke in patients with dengue. J Stroke Cerebrovasc Dis. 2010;19(3):253-256.

79. de Souza LJ, Martins AL, Paravidini PC, et al. Hemorrhagic encephalopathy in dengue shock syndrome: a case report. Braz J Infect Dis. 2005;9(3):257-261.

80. Liou LM, Lan SH, Lai CL. Dengue fever with ischemic stroke: a case report. Neurologist. 2008;14(1):40-42.

81. Seet RC, Lim EC. Dysarthria-clumsy hand syndrome associated with dengue type-2 infection. J Neurol. 2007;254(8):1129-1130.

82. Basu A, Chaturvedi UC. Vascular endothelium: the battlefield of dengue viruses. FEMS Immunol Med Microbiol. 2008;53(3):287-299.

83. Gupta A, Srinivasan R, Setia S, Soundravally R, Pandian DG. Uveitis following dengue fever. Eye (Lond). 2009;23(4):873-876.

84. Bacsal KE, Chee SP, Cheng CL, Flores JV. Dengue-associated maculopathy. Arch Ophthalmol. 2007;125(4):501-510.

85. Haritoglou C, Dotse SD, Rudolph G, Stephan CM, Thurau SR, Klauss V. A tourist with dengue fever and visual loss. Lancet. 2002; 360(9339):1070.

86. Teoh SC, Chee CK, Laude A, Goh KY, Barkham T, Ang BS; Eye Institute Dengue-related Ophthalmic Complications Workgroup. Optical coherence tomography patterns as predictors of visual outcome in dengue-related maculopathy. Retina. 2010;30(3):390-398.

87. Su DH, Bacsal K, Chee SP, et al; Dengue Maculopathy Study Group. Prevalence of dengue maculopathy in patients hospitalized for dengue fever. Ophthalmology. 2007;114(9):1743-1747.

88. Dussart P, Petit L, Labeau B, et al. Evaluation of two new commercial tests for the diagnosis of acute dengue virus infection using NS1 antigen detection in human serum. PLoS Negl Trop Dis. 2008;2(8):e280.

89. Gowri Sankar S, Dhananjeyan KJ, Paramasivan R, Thenmozhi V, Tyagi BK, John Vennison S. Evaluation and use of NS1 IgM antibody detection for acute dengue virus diagnosis: report from an outbreak investigation. Clin Microbiol Infect. 2012;18(1):E8-E10.

90. Blacksell SD, Bell D, Kelley J, et al Prospective study to determine accuracy of rapid serological assays for diagnosis of acute dengue virus infection in Laos. Clin Vaccine Immunol. 2007;14(11):1458-1464.

91. Nunes MR, Nunes Neto JP, Casseb SM, et al. Evaluation of an immunoglobulin M-specific capture enzyme-linked immunosorbent assay for rapid diagnosis of dengue infection. $J$ Virol Methods. 2011;171(1):13-20.

92. Araújo FM, Brilhante RS, Cavalcanti LP, et al. Detection of the dengue non-structural 1 antigen in cerebral spinal fluid samples using a commercially available enzyme-linked immunosorbent assay. $J$ Virol Methods. 2011;177(1):128-131.

93. Soares CN, Faria LC, Peralta JM, de Freitas MR, Puccioni-Sohler M. Dengue infection: neurological manifestations and cerebrospinal fluid (CSF) analysis. J Neurol Sci. 2006;249(1):19-24.

94. Webster DP, Farrar J, Rowland-Jones S. Progress towards a dengue vaccine. Lancet Infect Dis. 2009;9(11):678-687.
Research and Reports in Tropical Medicine

\section{Publish your work in this journal}

Research and Reports in Tropical Medicine is an international, peerreviewed, open access journal publishing original research, case reports, editorials, reviews and commentaries on all areas of tropical medicine, including: Diseases and medicine in tropical regions; Entomology; Epidemiology; Health economics issues; Infectious disease; Laboratory

\section{Dovepress}

science and new technology in tropical medicine; Parasitology; Public health medicine/health care policy in tropical regions; and Microbiology. The manuscript management system is completely online and includes a very quick and fair peer-review system. Visit http://www.dovepress. com/testimonials.php to read real quotes from published authors. 